\title{
Frontières
}

\section{Une décennie de publication sur la mort}

Frontières 1988-1998

\section{Renaud Santerre et Marie-Ève Carle}

Volume 12, numéro 2, printemps 2000

URI : https://id.erudit.org/iderudit/1074408ar

DOI : https://doi.org/10.7202/1074408ar

Aller au sommaire du numéro

Éditeur(s)

Université du Québec à Montréal

ISSN

1180-3479 (imprimé)

1916-0976 (numérique)

Découvrir la revue

Citer ce document

Santerre, R. \& Carle, M.-È. (2000). Une décennie de publication sur la mort :

Frontières 1988-1998. Frontières, 12(2), 80-86. https://doi.org/10.7202/1074408ar d'utilisation que vous pouvez consulter en ligne.

https://apropos.erudit.org/fr/usagers/politique-dutilisation/ 


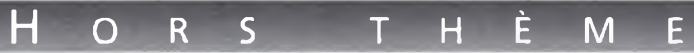

\section{UNE DÉCENNIE DE PUBLICATION SUR LA MORT FRONTIÈRES 1988 - 1998}

\author{
Renaud Santerre, \\ professeur au Département d'anthropologie \\ de I'Université Laval. \\ Marie-Ève Carle, \\ étudiante au baccalauréat en anthropologie \\ à l'Université Laval.
}

Seule revue francophone d'Amérique consacrée à l'étude de la mort, Frontières occupe le difficile créneau de la vulgarisation scientifique. Sa naissance en 1988 au sein du programme d'études interdisciplinaires sur la mort, à l'UQAM, lui a fait dans un premier temps profiter d'un statut quasi institutionnel, qui n'a été formellement reconnu qu'en 1994 et qui se trouve présentement remis en question.

Une décennie de publication sur la mort au Québec mérite qu'on s'y arrête un peu. La mort en effet est un thème par nature multidisciplinaire, en ce sens qu'aucune discipline déjà constituée ne peut se l'approprier et envisager d'en épuiser le contenu. Aussi l'étude de ce sujet doit-elle faire face à un défi de taille: celui de l'interdiciplinarité.

Le bilan proposé ici repose sur une analyse de contenu des dix premiers volumes (30 numéros) de la revue ${ }^{1}$, auxquels s'ajoutent les deux premiers numéros du volume 11 ; sur les évaluations périodiquement effectuées par des spécialistes externes, le Service des publications de l'UQAM et les organismes subventionnaires; sur le jugement personnel que peut porter un collaborateur de première heure qui participe au comité de rédaction.

\section{BRÈVE PRÉSENTATION}

C'est au printemps 1988 que Frontières paraît pour la première fois sous la forme d'un quadrimestriel dont le premier numéro de 68 pages $(8,5$ po $\mathrm{x}$ 11 po) est enclos sous une couverture cartonnée avec une bannière caractéristique, qui restera jaune pour les quatre premiers volumes. À compter du cinquième volume, la couleur variera avec chaque numéro.

Abondamment illustré, ce périodique voué à un sujet complexe et parfois périlleux se démarque de l'austérité habituelle des revues scientifiques, de format moindre, sans illustration et de plus grande épaisseur. Dès le départ, on vise le créneau de la vulgarisation scientifique, donc une clientèle plus nombreuse et diversifiée que ces dernières, non sans soulever des attentes plus disparates, voire contradictoires, et créer des problèmes particuliers au niveau du financement.

Le sous-titre originel, Revue d'information, de réflexion et de vulgarisation scientifique sur le mourir, la mort et le deuil, traduit bien l'orientation de cette revue. Les modifications du sous-titre en cours de route (vol. 2, no 3; vol. 4, no 1) et sa disparition à compter du volume 9 n'entraînent aucun changement d'orientation majeur ${ }^{2}$.

La périodicité originelle, trois numéros à thème par an-volume de 180 pages environ, demeure la même pendant la décennie jusqu'au changement majeur survenant avec le numéro 1 du volume 12. La revue devient alors semestrielle.
Le nombre de numéros passe de trois à deux par an tout en conservant sensiblement le même nombre de pages par volume. Les numéros restent à thème unique, mais sans exclure un article hors thème chaque fois.

Toute une série de thèmes ont déjà fait l'objet d'un, parfois deux numéros. Depuis quelques années, Frontières fait appel à un ou deux responsables de thème par numéro, qui se chargent, sous la responsabilité générale de la direction et du comité de rédaction de la revue, de proposer un thème, d'en définir les différentes facettes et de rechercher une variété de collaborateurs pour en traiter.

C'est le philosophe Fernand Couturier qui a présidé au lancement de cette revue et en a assuré la directionrédaction jusqu'au vol. 3 , no 2 , à l'été 1990. Il était déjà responsable à l'UQAM des programmes (diplôme de 2e cycle et certificat) d'études interdisciplinaires sur la mort. Avec l'anthropologue Renaud Santerre à l'Université Laval, il était également la cheville ouvrière d'un projet, malheureusement avorté du côté de Laval, de maîtrise conjointe LAVAL-UQAM en études interdisciplinaires de la mort.

Frontières est donc née à l'UQAM et reste l'un des fleurons de cette institution en matière de publication périodique. Juridiquement cette revue a toujours relevé directement et uniquement de l'UQAM. En témoigne la déclaration du 11 septembre 1987, par laquelle la mandataire de l'UQAM "déclare qu'à compter du 8 septembre 1987, la dite 
Université à titre de propriétaire, éditeur, publiera une revue périodique sous le nom: Frontières, revue d'information, de réflexion et de vulgarisation scientifique."

Le tirage de la revue a évolué de 2000 environ au départ à quelque 1500 exemplaires. Le millier d'abonnés des débuts s'est un peu réduit à la longue au gré des crises économiques et de la perte progressive, suite aux compressions budgétaires, des abonnements institutionnels. Si bien qu'aujourd'hui le nombre d'abonnés fluctue autour de 600 . Diversifiée, cette clientèle comprend des chercheurs en petit nombre, surtout des intervenants du réseau de la santé, des étudiants universitaires et collégiaux, des thanatopracticiens et un public cultivé qu'intéressent tous les phénomènes entourant la mort. À cette diversité du lectorat correspond la variété des contenus publiés dans la revue.

\section{CONTENU DE FRONTIÈRES}

La thématique de Frontières est le premier sujet à retenir l'attention dans une analyse de son contenu ${ }^{3}$.

\section{LISTE DES THĖMES}

On rappelle régulièrement en fin de chaque numéro, et ce à partir du volume 4 , les thèmes déjà abordés et l'on annonce le thème du numéro à venir ainsi que des numéros en préparation.

L'examen sommaire de la liste des thèmes traités depuis 1988 (voir page 96) permet de constater le retour de certains thèmes comme le suicide, le deuil, les rituels, bien que ces thèmes récurrents soient abordés chaque fois dans une perspective nouvelle. Plusieurs thèmes particuliers (enfance, adolescence, vieillesse; le féminin et maternité; la ville et la terre) pourraient se regrouper sous un thème plus général: la mort et les âges de la vie; féminité et mort; mort et environnement.

Certains thèmes, v.g. communautés culturelles, guerres, rites, cimetières, se prêtent mieux à une approche plutôt sociologique ou anthropologique, d'autres à une perspective surtout historique (civilisations perdues?) ou plutôt psychologisante. D'autres thèmes (soins palliatifs) font une large place à l'intervention.

Plus qu'un essoufflement après une décennie de publication, le retour de certains thèmes semble indiquer, après un premier tour de piste exploratoire, le besoin d'approfondir certaines questions fondamentales ou d'actualiser les problématiques.

\section{ILLUSTRATIONS}

La vulgarisation scientifique, que le jargon des administrations appelle "transfert des connaissances», n'est pas un objectif facile à atteindre. Sans rien sacrifier de la scientificité du contenu, il faut viser un public cultivé et ouvert sans doute, mais que rebuteraient une technicité excessive et un style par trop hermétique ou ésotérique. $\mathrm{Ce}$ public veut pouvoir feuilleter sa revue et lire tout un article dans le métro ou dans la salle d'attente de son dentiste. D'où le recours par Frontières, dans un format manipulable, à une multiplicité de textes différents, plutôt courts et abondamment illustrés. Les illustrations sont une marque de commerce de Frontières. La page couverture en particulier donne le ton et, en quelque sorte, annonce les "couleurs", même si les pages intérieures se limitent au noir et blanc.

Suivant les numéros, l'espace total occupé par les illustrations dans la revue varie en pourcentage d'un maximum de 25 (vol. 5, no 1) à un minimum de 3\% (vol. 8, no 1). Jusqu'au volume 6, l'espace illustré est supérieur à 10\%, mais il descend, à compter de 6,3, en dessous de la barre des 10\%, qu'il ne franchit jamais plus. On assiste donc à une diminution des illustrations par rapport aux textes au cours de la décennie.

Passant de l'espace occupé au nombre d'illustrations par numéro, on constate également une grande variabilité: de 32 à 52 jusqu'au vol. 6, no 2, et de 17 à 34 plus récemment. La moyenne d'illustrations par numéro, de 33 qu'elle est en général $(1068 * / 32)$ pendant toute la période connaît elle aussi une chute de 39 (moyenne des 16 premiers numéros) à 28 (moyenne des 16 derniers).

On note aussi des variations d'une catégorie d'illustrations à l'autre. Les photographies représentent $55 \%$ du total des illustrations et ont tendance à augmenter comparativement aux dessins, qui occupent le second rang (32\%) du total, mais voient leur fréquence relative diminuer avec le temps.

Inexistantes jusqu'au volume 4 , les reproductions d'oeuvre d'art tendent à occuper une place plus significative. En nombre pendant toute la période, les reproductions revêtent à peu près la même importance ( $+6 \%$ chacun) que les tableaux chiffrés et les graphiques.

\section{ESPACES PUBLICITAIRES}

Le recours à la publicité dans Frontières apparaît marginal: à peine une dizaine d'espaces publicitaires par année-volume. Jusqu'au volume 9, le programme d'études interdisciplinaires sur la mort de l'UQAM et l'Association des diplômés de ce programme, l'ADEM, étroitement reliée à Frontières, semblent réserver à leur promotion l'extérieur de la dernière page couverture, qui reste vierge par la suite. Comme si la revue hésitait à encaisser les revenus que rapporte cet espace publicitaire le plus payant.

Récemment on lorgne plus facilement du côté des pages de garde intérieures pour abriter les annonces, assez bien faites d'ailleurs, d'entreprises funéraires comme le Cimetière Notre-Damedes-Neiges et même Alfred Dallaire (vol. 11, no 3).

\section{COMPTES RENDUS}

De tous les textes à caractère thanatologique, les Comptes rendus dans Frontières représentent peut-être la rubrique la plus fournie, celle qui manifeste la plus grande vitalité et témoigne d'un effort soutenu de vulgarisation scientifique. Régulièrement le public cultivé se trouve ainsi mis au courant de la production thanatologique.

Le nombre de comptes rendus classiques sur le thème des numéros croît sûrement de 2 (vol. 1, no 1) à 14 (vol. 10, no 3) avec une moyenne d'environ 6 comptes rendus par numéro. C'est un complément obligé aux articles qui traitent du thème.

À ranger dans cette catégorie de textes auxiliaires de la plus grande utilité, il faut mentionner les bibliographies et filmographies thématiques, les listes de nouvelles parutions et la très moderne chronique informatique De l'éternité @ l'internité.

\section{CATÉGORISATION DES TEXTES}

Le Sommaire qui paraît en tête de chaque numéro classe l'ensemble des textes publiés dans une grande variété de catégories, dont le nombre et l'intitulé évoluent avec le temps sans que la rationalité de cette répartition ni l'étanchéité des catégories soient toujours évidentes.

Mis à part le Sommaire, l'Éditorial, la Présentation du numéro et les chroniques bibliographiques déjà mentionnées, on trouve des Articles de fond, des Notes de recherche, des Dossiers, des Témoignages, des Interventions, des chroniques d'humour (disparues à la longue), d'Art et de poésie, des Points de vue, des Regards, etc. La caractéristique de tous ces textes de longueur et de style différent, c'est de traiter de la mort sous un angle particulier et de provenir de la plume originale d'un auteur singulier, dont la discipline et l'appartenance institutionnelle sont généralement connues. 
Sur 32 numéros en près de onze années, on constate que 18 textes, tous publiés dans les trois premiers numéros du volume 1, entrent dans la catégorie Dossier; qu'un Éditorial coiffe chacun des 32 numéros, alors que seulement 7 , surtout au début, se méritent un texte de Présentation; la catégorie Article (180 textes) est la plus régulièrement représentée pendant toute la période, sa part de l'ensemble total s'établissant à $20 \%$ et variant entre le huitième et le tiers de la matière publiée dans un numéro.

Art et poésie fleurissent au départ de la revue (près de $10 \%$ de la matière publiée) et meurent en 1994. Est-ce sous le coup de dures contraintes économiques? Il en va de même de la catégorie Humour qui disparaît encore plus tôt (1992).

La catégorie Justice s'évanouit dès le troisième volume, sauf fantomatique réapparition en 1993. Les Témoignages, nombreux au début, se maintiennent jusqu'en 1995, totalisant 42 expressions en 7 ans avant de disparaître complètement. La catégorie Point de vue prend la relève depuis, si bien qu'à elles deux ces catégories représentent un total de 80 insertions en près de onze ans.

La catégorie Intervention est encore plus fournie et régulière pour un total de 122 textes pendant toute la période. C'est $13,5 \%$ de la matière publiée. De même importance $(124$ textes $=13,8 \%)$ et régularité se trouve la catégorie Recherche. Les catégories Courrier, ADEM et autres sont résiduaires. On a déjà fait état de la catégorie Lecture $(21,4 \%)$ représentée par les comptes rendus.

L'essentiel de la matière originale publiée dans Frontières se retrouve ainsi dans les catégories suivantes qu'il convient de regrouper: Articles (20\%), Recherche $(13,8 \%)$ et Intervention $(13,5 \%)$.

\section{COLLABORATEURS}

Le bassin des auteurs de textes à Frontières est large et leur provenance fort diverse.

\section{DISCIPLINES}

Quelque 500 collaborateurs ont signé des textes à Frontières. Parmi les 400 environ dont la discipline ou le champ d'études est connu, le Tableau I range les 20 premiers par ordre de fréquence, encore qu'un regroupement plus inclusif de disciplines grossirait certaines catégories et modifierait peut-être le classement: par exemple psychanalyse avec psychologie, psychiatrie avec médecine,

\section{TABLEAU I}

RÉPARTITION PAR DISCIPLINE OU CHAMP D'ÉTUDES DES COLLABORATEURS FRONTIËRES, $1988-1998$

Discipline
ou champ d'études

\begin{tabular}{lcc}
\hline $1^{*}$ Psychologie & 94 & 19.3 \\
\hline $2 *$ Médecine & 42 & 8.6 \\
\hline $3 *$ Soins infirmiers & 35 & 7.2 \\
\hline $4^{*}$ Études sur la mort & 33 & 6.8 \\
\hline $5^{*}$ Littérature et poésie & 27 & 5.5 \\
\hline $6 *$ Sciences des religions & 25 & 5.1 \\
\hline $7 *$ Travail social & 24 & 4.9 \\
\hline $8^{*}$ Éducation & 20 & 4.1 \\
\hline $9 *$ Sexologie & 16 & 3.3 \\
\hline $10^{*}$ Anthropologie et ethnologie & 16 & 3.3 \\
\hline $11^{*}$ Arts (musique, peinture, danse) & 15 & 3.1 \\
\hline $12^{*}$ Droit & 14 & 2.9 \\
\hline $13^{*}$ Philosophie & 14 & 2.9 \\
\hline $14^{*}$ Communications & 13 & 2.7 \\
\hline $15^{*}$ Sociologie et science politique & 11 & 2.3 \\
\hline $16^{*}$ Psychanalyse & 10 & 2.0 \\
\hline $17^{*}$ Histoire & 9 & 1.8 \\
\hline $18^{*}$ Théologie & 9 & 1.8 \\
\hline $19^{*}$ Administration & 8 & 1.6 \\
\hline $20^{*}$ Journalisme & 7 & 1.4 \\
\hline
\end{tabular}

éthique avec philosophie, pastorale avec théologie, etc. Le rangement des auteurs par catégorie tient compte de la première identification disciplinaire fournie par l'auteur lui-même. Nettement en tête vient la psychologie avec 94 collaborateurs de cette discipline, soit près de $20 \%$ du total. Un collaborateur sur cinq pratique la psychologie et intervient auprès de ses clients et lecteurs dans l'une ou l'autre branche de cette discipline multiforme.

Assez curieusement les huit catégories qui suivent par ordre décroissant couvrent moins une discipline qu'un champ d'études ou d'intervention, comme la médecine, les soins infirmiers, le travail social, l'éducation et même la sexologie, où interviennent de concert plusieurs disciplines formelles. Les études sur la mort sont par nature interdisciplinaires, même si une pluralité de psychologues y agissent comme partout ailleurs.

Les disciplines étroitement constituées, qu'on retrouve clairement identifiées sur les campus universitaires, comme l'anthropologie, le droit, la philosophie, la sociologie, l'histoire et la théologie, suivent loin derrière les champs d'intervention. À croire que la revue Frontières serait plutôt psychologisante et livrée aux mains des interventionnistes.

\section{RATTACHEMENT}

La liste des institutions auxquelles sont rattachés les collaborateurs de Frontières vient préciser et nuancer cette interprétation, ainsi qu'en témoigne le Tableau II.

Deux catégories d'institutions se partagent la quasi totalité des collaborateurs dont l'affiliation institutionnelle est connue; près du tiers des collaborateurs ne se rattachent ouvertement à aucune institution. C'est l'université qui fournit le plus fort contingent $(235=$ $48 \%$ ) de collaborateurs. Un collaborateur sur deux appartient au monde de l'éducation collégiale et universitaire. Quelque 89 collaborateurs (18\%) oeuvrent dans le domaine de la santé, principalement dans les hôpitaux et un peu dans les CLSC.

L'UQAM (25\%), l'Université de Montréal (11\%) et l'Université Laval (4\%) fournissent les plus forts contingents de collaborateurs universitaires, qui se ventilent entre 26 institutions. Les dix universités le mieux représentées sont canadiennes. L'éparpillement est surtout manifeste entre 16 universités européennes et américaines, qui ne sont présentes que par un seul collaborateur chacune.

Du côté hospitalier, 29 centres sont actifs. Viennent en tête de liste l'Hôpital Sainte-Justine avec $2 \%$ du grand total de collaborateurs, le Royal Victoria $(1,4 \%)$, 


\section{TABLEAU II}

RÉPARTITION DES INSTITUTIONS AUXQUELLES SONT RATTACHÉS LES COLLABORATEURS

FRONTIERES, 1988 - 1998

\section{Institutions}

Nombre de collaborateurs

Proportion (\%)

\begin{tabular}{lrr}
\hline Universités & 235 & 48 \\
\hline Collèges & 11 & 2 \\
\hline Hôpitaux & 70 & 14 \\
\hline CLSC et apparentés & 19 & 4 \\
\hline Autres et indéterminés & 154 & 32 \\
\hline Total & & \\
\hline
\end{tabular}

l'Hôpital pour enfants de Montréal $(1,2 \%)$ et l'Hôpital Notre-Dame $(1,2 \%)$. Là aussi les institutions montréalaises prédominent nettement et le secteur hospitalier international ne figure ici que par deux hôpitaux de Paris. Cette concentration montréalaise des collaborateurs, dont $25 \%$ se retrouvent à I'UQAM, marraine de la revue sans avoir de faculté de médecine ni d'hôpital universitaire, n'a rien d'étonnant. Le contraire serait même un peu inquiétant. N'empêche que la diversification tant institutionnelle que disciplinaire des collaborations est un objectif toujours à poursuivre et jamais atteint par une revue unique en son genre qui vise l'internationalisme. Les organismes subventionnaires en tiennent un compte rigoureux et forcent les revues candidates à jouer dans les ligues majeures.

Près de 500 auteurs au cours de la dernière décennie ont donc collaboré à Frontières en fournissant un ou des alphabétique du Tableau III. textes dans l'une ou l'autre catégorie, soit un article de fond, un poen tous ces collaborateurs en fonctio décroissante du nombre d'interventions écrites dans la revue fait apparaître en à regrouper en une seule les trois catégories Articles, Recherche et Intervention en totalisant le nombre de pages
N'ont été retenus, dans le Tableau III, que les auteurs d'au moins deux textes totalisant 10 pages ou plus et entrant dans les trois catégories: Article, Recherche et Intervention. Parmi ces 19 auteurs réguliers et prolifiques figurent 9 professeurs de l'UQAM, 5 d'autres universités et 4 spécialistes des hôpitaux; 5 psychologues, 3 religiologues, 3 anthropologues et 2 sexologues. On y retrouve également 4 directeurs et/ou rédacteurs de la revue.

À l'examen du tableau III, on constate le maintien aux premiers rangs des collaborateurs réguliers et prolifiques de trois des quatre ténors de l'UQAM déjà identifiés: Luce Des Aulniers, Joseph Josy Lévy et Éric Volant. La quatrième disparaît de la liste parce que ses contributions nombreuses se rangent parmi les comptes rendus et les chroniques bibliographiques ou filmographiques, que ses fonctions d'agente d'information l'incitaient à produire régulièrement. Les trois leaders sont suivis de Lucie Fréchette, psychologue de l'UQAH, de l'anthropologue français Louis-Vincent Thomas, de Suzanne Mongeau, travailleuse sociale, de la sexologue de I'UQAM, Denise Badeau, du psychologue Paul Frappier, également de I'UQAM, et de Fernand Couturier, directeur-fondateur de la revue à l'UQAM.

\section{DIRECTION ET/OU RÉDACTION}

La première page, recto et verso, de la revue fournit habituellement, outre le sommaire du numéro, une abondance d'information sur les collaborateurs, la gestion, la rédaction et la direction de la revue.

TABLEAU III

LISTE ALPHABÉTIQUE DES PRINCIPAUX COLLABORATEURS

FRONTIËRES 1988 - 1998

Collaborateurs

Badeau Denise

Bourgeault Guy

Couturier Fernand

De Montigny Johanne

Des Aulniers Luce

Deschamps Danièle

Douesnard Suzanne

Frappier Paul

Fréchette Lucie

Hétu Jean-Luc

Larouche Jean-Marc

Lévy Joseph Josy

Miron Thérèse

Mongeau Suzanne

Saint-Arnaud Jocelyne

Santerre Renaud

Thomas Louis-Vincent

Viau-Chagnon Michèle

Volant Éric

\begin{tabular}{llr} 
Disciplines & Institutions & Textes \\
\hline Sexologie & UQAM & \\
\hline Éthique & U.de Montréal \\
\hline Philosophie & UQAM \\
\hline Psychologie & H. Royal Victoria \\
\hline Anthropologie & UQAM \\
\hline Psychologie & Suisse \\
\hline Psychologie & H. Ste-Justine \\
\hline Psychologie & UQAM \\
\hline Psychologie & UQAH \\
\hline Gérontologie & UQAM \\
\hline Théologie & U.St-Paul \\
\hline Sexologie & UQAM \\
\hline Religiologie & UQAM \\
\hline Travail social & UQAM \\
\hline Religiologie & UQAM \\
\hline Anthropologie & U. Laval \\
\hline Anthropologie & Sorbonne \\
\hline Soins infirmiers & Hôpital pour enfants \\
\hline Religiologie & UQAM \\
\hline
\end{tabular}

\begin{tabular}{rrr} 
es & Pages & Rang \\
\hline 6 & 20 & 7 \\
\hline 2 & 11 & 17 \\
\hline 5 & 11 & 9 \\
3 & 14 & 13 \\
18 & 93 & 1 \\
\hline 2 & 10 & 18 \\
\hline 2 & 11 & 16 \\
\hline 5 & 14 & 8 \\
\hline 6 & 29 & 4 \\
\hline 4 & 12 & 12 \\
\hline 3 & 11 & 15 \\
\hline 12 & 46 & 2 \\
\hline 4 & 16 & 10 \\
\hline 5 & 25 & 6 \\
\hline 3 & 12 & 14 \\
\hline 2 & 10 & 19 \\
\hline 5 & 29 & 5 \\
\hline 4 & 13 & 11 \\
\hline 7 & 30 & 3 \\
\hline
\end{tabular}


Le comité de rédaction, parfois intitulé comité consultatif et d'orientation, manifeste une certaine stabilité de composition sans qu'on puisse préjuger de l'identité du rôle joué par un tel comité sous les directions successives de la revue. Certains membres, comme Suzanne Mongeau et Renaud Santerre, y siègent depuis les débuts, mais souvent, en particulier entre la première et l'actuelle direction, de façon lointaine, voire purement nominale. C'est sous la direction de Fernand Couturier et celle de Luce Des Aulniers surtout que le comité de rédaction a joué pleinement son rôle consultatif d'orientation de la revue. Luce Des Aulniers est effectivement présente à la revue depuis les débuts.

Le comité de rédaction de 1998-99 se composait de quinze membres en provenance de l'UQAM (5), de l'Université de Montréal (3), de l'Université Laval (2) et de diverses institutions (5) reliées à la santé et intéressées à tous les phénomènes qui entourent la mort. Ce comité s'est réuni six fois au cours de l'année, a choisi les thèmes et responsables des numéros à venir, examiné l'avant-projet du numéro en préparation et procédé à l'évaluation critique de chaque numéro après parution. Ses avis circonstanciés ont toujours guidé la direction actuelle dans l'accomplissement de sa tâche.

Le titre de rédacteur apparaît parfois au sommaire, dissocié du comité de rédaction et porté par un seul individu, qui le cumule souvent, comme c'est le cas présentement, avec le titre de directeur. Voilà une de ces ambiguïtés de nomenclature qui perdurent à Frontières sans accroître d'une once son efficacité ou son rayonnement ${ }^{4}$.

C'est finalement au niveau de la direction de la revue que l'analyse de contenu sur une période de près de onze années révèle les plus grandes fluctuations et le plus sérieux problème: celui de la succession et de la continuité.

Le départ du directeur-fondateur Fernand Couturier en 1990, après la parution du vol. 3, no 1 , crée un vide que ne vient combler ni une direction collégiale des membres du "comité de direction et de rédaction" ni le recours à un "responsable du numéro", Denis Savard pour le vol. 3, no 2, et Joseph Josy Lévy pour le vol. 3, no 3. C'est seulement à partir du vol. 4, no 1, qu'Éric Volant succède réellement à Fernand Couturier au poste de directeur de la revue, qu'il occupera pendant plus de trois ans (10 numéros) pour le quitter à l'occasion de sa dernière année sabbatique avant sa retraite.
S'installe de nouveau un intérim sous la forme d'un(e) responsable de numéro, Jocelyne Saint-Arnaud, qui devient rapidement directrice après avoir été rédactrice en chef; elle avait accédé à ce "poste» particulier, sous la direction de Éric Volant, après Denis Savard, puis Guy Labelle. Au sortir du directorat de Jocelyne Saint-Arnaud (trois numéros du vol. 7 , no 2 au vol. 8 , no 1 ), nouvelle valse à la direction, qui se scinde en quatre (vol. 8, no 2): responsable de numéro, directeur scientifique, rédactrice en chef et directeur administratif. Cette démultiplication des directions perdure jusqu'au retour d'Éric Volant (vol. 9, no 3) à une direction unique, qui, comme dit le poète, "vivra ce que vivent les roses, l'espace d'un matin" (deux numéros).

C'est alors, à partir du volume 10 , qu'intervient l'administration de l'UQAM qui sert à Luce Des Aulniers l'alternative suivante: prendre la direction de Frontières ou en accepter la disparition ${ }^{5}$. Malgré le dynamisme de la directricerédactrice actuelle, le problème demeure entier puisque, au terme de son triennat à la parution du vol. 12 , no 2 , la question reste toujours celle d'Hamlet: «To be or not to be».

Ici se clôt l'analyse de contenu proprement dite de Frontières pour laisser place aux évaluations externes de cette décennie de publication sur la mort et pour aborder les problèmes de gestion administrative, technique et financière qui handicapent et menacent même la survie de la revue.

\section{ÉVALUATIONS EXTERNES}

Au-delà d'une analyse de contenu, à caractère plutôt objectif, et des évaluations internes, qui risquent toujours d'être entachées de parti pris, il importe de rechercher une évaluation critique indépendante de cette revue particulière.

On peut trouver ce jugement d'ensemble indépendant d'abord dans le rapport d'évaluation (trois évaluateurs externes) requis par l'UQAM en $1994 \mathrm{au}$ moment d'accorder sa reconnaissance institutionnelle à la revue et dans les évaluations qui fondent en 1998 l'octroi des subventions du CRSHC et du FCAR.

\section{ÉVALUATIONS DE 1994}

"La revue Frontières, de l'avis du premier évaluateur, comporte un aspect tout à fait original: le nombre de ses rubriques. Elle veut rejoindre à la fois les scientifiques, les professionnels et le grand public. C'est là, à mon avis, sa richesse et sa faiblesse. Peut-elle maintenir tous ses objectifs en même temps?
[...] En général, je dirais que la revue Frontières est très bien adaptée au public professionnel et au grand public. [...] J'apporterais des réserves concernant le caractère scientifique de la revue. À mon avis, le secteur Recherche en est le secteur le plus pauvre.»

Le deuxième évaluateur reconnaît que, "dans l'ensemble, la revue répond assez bien au critère de la vulgarisation scientifique, même si la qualité moyenne n'est pas constante parmi les quatre numéros analysés».

D'emblée, le troisième lecteur externe souligne que "Frontières occupe un créneau de recherche, de réflexion et d'intervention qui mérite largement d'être soutenu». [...] "Malgré quelques faiblesses, la revue constitue un très bon produit. Pour peu que sa direction maintienne le cap actuel, il me semble que Frontières est engagée sur la voie prometteuse d'une publication qui accepte de relever le difficile défi de s'adresser à un public varié.»

"Frontières doit se souvenir, ajoute-til, qu'elle est d'abord une revue universitaire, ce qui veut dire, à mes yeux, une revue où s'exerce et se cultive une tradition de critique à l'égard des courants de pensée dominants dans une société. Certes, cette tradition pèche souvent par académisme et elle se vautre trop facilement dans la langue de bois que ne traverse plus aucun courant chaud; aussi convient-il de la renouveler et de l'adapter à un public plus large, ce que Frontières réussit d'ailleurs à faire».

Ces jugements d'ensemble, somme toute assez favorables, se trouvent confirmés quatre ans plus tard par les évaluations des conseils subventionnaires.

\section{ÉVALUATIONS DE 1998}

Accordant à Frontières la cote $\mathrm{B}+$, qui justifie une subvention, le Fonds FCAR «estime que Frontières occupe une niche thématique bien spécifique qui, tel qu'en témoigne la diffusion de cette récente revue, répond à un besoin du milieu. Cependant, il s'attend à ce que la direction fournisse les efforts nécessaires pour assurer une qualité égale parmi les articles publiés.» "Exceptionnelle revue de transfert", note un évaluateur du Fonds.

Du côté du Conseil de recherches en sciences humaines du Canada, le comité d'aide aux revues de recherche et de transfert émet le commentaire suivant: "Le comité considère que cette revue multidisciplinaire est intéressante, et que dans l'ensemble, on y traite de sujets importants liés aux études sur la mort. 
Selon le comité, on aurait avantage à rehaussser la qualité scientifique des articles soumis afin de mettre en relief le rôle unique et significatif de cette revue de transfert. Le comité estime que l'arrivée de madame Des Aulniers en tant que rédactrice de la revue permettra à la revue de s'orienter dans cette voie».

De 1994 à 1998, les évaluations externes dénotent une amélioration de la qualité scientifique de Frontières, et son statut de revue de transfert, enfin reconnu, la rend admissible au support des organismes subventionnaires. Par contre la multidisciplinarité de ses collaborateurs la coupe d'un plein financement par le Conseil de recherches en sciences humaines. C'est ce qui complique la gestion d'une telle revue et, à la longue, risque de lui coûter la vie. À toujours être assis entre deux ou plusieurs chaises, il est inévitable de se retrouver le derrière au sol!

\section{GESTION ADMINISTRATIVE, TECHNIQUE ET FINANCIĖRE}

La publication d'une revue pose des problèmes de gestion à la fois administrative, technique et financière, qu'il convient d'aborder séparément.

\section{ADMINISTRATION}

L'administration d'une revue comme Frontières repose sur le travail effectif, bénévole et rémunéré, d'une équipe restreinte constituée autour de la direction de la revue. Au cours du dernier triennat, Luce Des Aulniers s'est appuyée sur un comité de gestion de cinq membres, bénévoles, dont certains siégeaient également au comité de rédaction, et sur le travail rémunéré d'une secrétaire d'édition, recrutée dans le cadre régulier des personnels de l'UQAM. Point n'est besoin d'énumérer toutes les tâches à accomplir par ce comité de bénévoles et par ce personnel spécialisé, qui en réalité occupe, après la direction, la deuxième fonction en importance à la revue.

La difficulté du renouvellement à la direction de la revue provient en bonne part des coupures progressives imposées au personnel rémunéré pour tenter de réduire d'un tiers le budget annuel des dépenses, qui était de 75000 \$ en 198889. Le poste d'agente d'information fourni par le CIREM est disparu avec ce dernier et l'on a réduit de 4 à 3 jours/semaine le temps de secrétariat, dont Frontières doit assumer entièrement les frais.

Aucune revue ne peut survivre sans secrétariat rémunéré: autrement c'est la direction qui se voit accablée de toutes les tâches. Et quand la direction profes- sorale se fait refuser dans son institution tout dégrèvement de cours ou, ce qui revient au même, est forcée de compenser ce dégrèvement à même le budget de la revue, il n'y a plus rien à faire ${ }^{6}$. N'importe qui tomberait malade dans ces conditions!

\section{PRODUCTION}

La production d'une revue suppose un ensemble d'opérations techniques qui passent par le graphisme, la composition, la mise en page, l'impression, la reliure jusqu'à l'expédition. À force d'économies, on a réduit le coût de ces opérations techniques, de $35000 \$$ qu'il était en 1988-89, à un peu plus de 20000 \$ par année. Sans compter les services gratuits fournis par l'UQAM sous forme de gestion des abonnements et des ventes, de gestion des comptes, des frais de poste, d'emballage et de manutention pour les expéditions. La direction de l'UQAM en 1998 estimait à $16000 \$$ cette aide gratuite annuelle consentie à Frontières du fait de son statut de revue institutionnelle.

\section{FINANCEMENT}

De 75000 \$ environ à sa création en 1988-89, le budget annuel de Frontières a été ramené aux alentours de 50000 \$ par année sans compter les coûts réels du support institutionnel. C'est un budget comparable à celui de revues scientifiques du même genre. Le coût des personnels impliqués, y compris le dégagement professoral de la direction, voisine les $30000 \$$.

Les revenus propres de la revue proviennent des abonnements et de la vente au numéro. Plus que les revues proprement scientifiques, une revue de vulgarisation comme Frontières obtient une part importante de ses revenus de la vente au numéro. Encore faut-il soigner la distribution et multiplier les points de vente.

En gros, Frontières tire plus du tiers de ses recettes des abonnements et de la vente, un tiers des subventions et le quart du support institutionnel. La deuxième de ces sources, plus récente, revêt un caractère plus aléatoire et dépend de son statut institutionnel. C'est dire que la reconnaissance de Frontières comme revue institutionnelle par l'UQAM est essentielle à sa survie.

\section{ÉPILOGUE \\ (EN GUISE D'ÉPITAPHE?)}

Le couperet est tombé. Sous la forme d'une décision du Comité des publications de l'UQAM de retirer à Frontières son statut de revue institutionnelle.
Dans une lettre adressée le 8 mai 2000 à la directrice intérimaire, la vice-rectrice à la recherche de l'UQAM statue: «En l'absence de réponses satisfaisantes aux demandes répétées des dernières années, les membres du Comité (des publications) ont décidé à l'unanimité de retirer à Frontières son statut de revue institutionnelle, catégorie "transfert», soit à compter du 31 mai 2001. Le Comité a laissé trois années aux membres de la rédaction de la revue pour trouver un remplaçant ou une remplaçante à la direction de Frontières. Les professeurs de l'UQAM ont tous refusé de poser leur candidature à titre de directeur scientifique, ne serait-ce que pendant deux ans pour relayer la directrice sortante, madame Luce Des Aulniers. De la même manière, les professeurs de Laval et de Montréal qui font partie du comité de rédaction de Frontières ont aussi décliné l'offre. Les membres du Comité des publications estiment qu'il serait irresponsable de la part de l'UQAM de cautionner encore longtemps la revue sur des bases aussi aléatoires".

Le mandat de Luce Des Aulniers à la direction de Frontières s'est donc terminé le 31 mai 2000 après trois ans de dur labeur. Du même coup une partie des 15 membres du comité de rédaction qu'elle avait réunis autour d'elle quittent le navire, s'ils n'avaient déjà pris le large. On laisse à une directrice intérimaire, déjà engagée dans une prochaine retraite, la charge "d'assumer la publication des trois numéros à venir» 7 . Et de liquider l'entreprise?

À très court terme, peut-être est-ce le programme d'études interdisciplinaires sur la mort, ou ce qu'il en reste depuis les coupures de 1997, qui est menacé de disparition? Qu'en pensent les membres de l'ADEM? L'espérance de vie de ce champ d'études très prometteur n'aurait guère dépassé vingt ans. Pouvait-il en être autrement quand on connaît la difficulté fondamentale d'une pratique véritable de l'interdisciplinarité? À titre d'observateur et d'acteur sur la scène universitaire au Québec, c'est la question que se pose en terminant le premier auteur de ce bilan.

À y bien regarder, la question de la mort subit le même sort que celui de la vieillesse dans la dizaine d'universités francophones d'Amérique du Nord: tous les programmes interdisciplinaires d'études sur ces deux thèmes d'importance vitale ont connu un essor remarquable dans les années 1980, mais subissent la même déchéance fatale au cours de la décennie suivante à cause justement de leur interdisciplinarité. Parce 
qu'ils n'avaient nulle part pignon sur rue, aucune unité de rattachement n'a pu les protéger des coupures et leur assurer les ressources financières, secrétariales et surtout professorales sans quoi on ne peut maintenir en vie un programme majeur d'études et produire une revue de qualité.

Par une soirée de gala avec piano, danse et chants d'opéra sur un thème "À la frontière des sortilèges" le samedi 31 octobre 1998, la revue Frontières a célébré son dixième anniversaire d'existence. À quels autres sortilèges eût-il fallu recourir pour assurer son développement au cours d'une deuxième décennie?

\section{Notes}

1 La majeure partie de cet article, consacrée à l'analyse de contenu proprement dite, a été rédigée à l'automne 1999. C'est en juin 2000 qu'on a complété l'analyse des évaluations, l'examen de la gestion administrative, technique et financière et la rédaction de l'épilogue.

2 N.D.L.R.: Frontières se présente maintenant comme une "revue québécoise d'information, de recherche et de transfert de connaissances en études sur la mort». Sans effectivement que ce changement de sous-titre ait introduit une modification majeure, il reste que l'exigence à la fois de scientificité et d'accessibilité des textes s'est accrue.

3 N.D.L.R.: d'évidence, il ne s'agira pas ici d'une analyse de contenu telle qu'on l'entend en recherche qualitative, portant sur des récits littéraires, de vie, de pratique, etc. Ainsi, par exemple, une analyse des sous-thèmes traités dans chaque numéro est impossible; toutefois le terme "analyse de contenu» est accrédité pour le type de description ici présenté.

4 N.D.L.R.: la direction de la revue veille au maintien de la qualité scientifique et à l'orientation de la revue, coordonne et anime le travail des équipes et individus, assure les relations publiques, les consultations, les demandes de subvention, la gestion du personnel, etc. ; la direction assume in finale les décisions sur la publication et travaille étroitement avec la rédaction et les responsables tout au long du numéro ; la rédaction, toujours sous la responsabilité de la direction, travaille directement sur le contenu de la revue et plus particulièrement l'évolution des textes et des rapports avec les auteurs. Rédaction et direction sont présentes lors de la phase de production.

5 N.D.L.R.: précision: devant le vacuum effectivement fort périlleux pour la revue, il allait de soi qu'une professeure membre du Comité de rédaction prenne le relais à la direction, de son plein gré.

6 N.D.L.R.: un organisme subventionnaire externe prévoit ce dégrèvement: il arrive que les revues l'emploient autrement, par obligation, ce qui fut parfois le cas. Un dégrèvement d'enseignement fut attribué de manière exceptionnelle par l'UQAM, en 1997.

7 N.D.L.R.: dans les faits, il s'agit des deux numéros du volume 13 , déjà avancés.

\section{Catherine Cyssau (dir. publ.) L'entretien en clinique}

Paris, In Press Éditions, 1998, 345 pages.

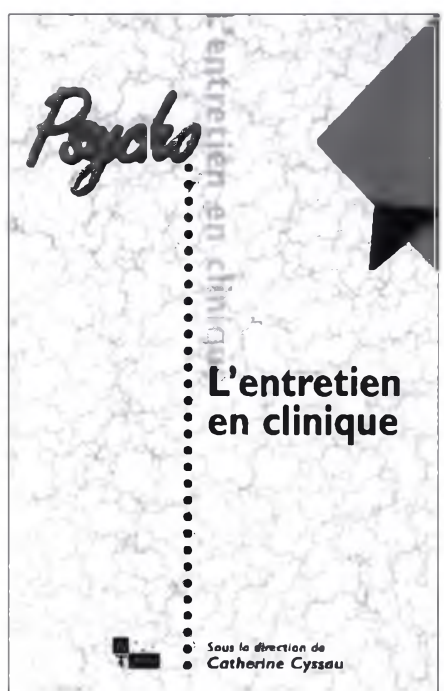

Le terme "entretien» connaît bien des infortunes du fait qu'il soit devenu un mot-valise. Ici, il est sondé avec une rigueur remarquable sous la multitude de prismes de la clinique du psychopathologique. C'est donc tout à fait bienvenu dans l'examen du phénomène de la peur, serti d'angoisse. Or, trois critères sont apposés et se répercutent au long des quelques vingt-deux courts et bien corsés chapitres de l'ouvrage (avec tout autant de collaborateurs): 1) Quels sont les modèles ou présupposés théoriques influençant la pratique de l'entretien?; 2) Quelles sont les modalités (parfois successives) visées par l'entretien?; 3) À la croisée de l'intrapsychique et de l'intersubjectif, quelles sont les formations psychiques à l'œuvre pour le patient, et à quels types d'interactions donnent-elles lieu? C'est en tenant compte de ces trois énoncés que le clinicien tourne le dos à la technique-prêt-à-porter, "qui sied mal à la parole dès lors qu'elle véhicule, avec tout le reste de l'expression et le mode de présence physique, émotionnelle, du patient à l'entretien, les processus psychiques et l'individuation symptomatique propre à chacun.» (p. 15).
Et là, les gourmets peuvent se mettre à table. Mais comment donner une idée fidèle du menu? Puisque chaque auteur admet d'emblée la complémentarité, voire l'imbrication des modèles de référence, le tout généralement transmis avec une grande clarté, ces aspects ne seront pas répétés. Plutôt que de présenter un survol global, on présentera, mi-arbitrairement, mi-«en questionnements réflexifs» ( $p$. 15) quelques extraits de chaque chapitre, dans la mire du thème de ce numéro de Frontières, concentrés alors plus en première partie.

1- Perspectives théoriques

"La parole et le langage en psychanalysen: toute mention d'un malaise serait sous-tendue par la croyance en la toute-puissance des idées, ainsi qu'en celle de l'interlocuteur, rêvant à l'éternité de l'inscription comme de la résolution. «ll semble que la transmutation liée au surgissement d'un sens imprévisible soit d'autant plus féconde que la parole est d'emblée reçue dans la stratification de ses dimensions: plainte corporelle, séduction, référence à un principe de légalité, récit établissant un passé, autant de niveaux s'offrant à être subvertis par l'effet d'une rencontre verbale. L'effet de sens interviendra nécessairement en prenant le visage de l'hôte indésirable ou du moins inattendu.» (p. 32).

"Les modèles de communication psychanalytique" renvoient quant à eux aux champs intercroisés de l'intrapsychique - plus à distance de l'expérience - et de l'interpersonnel. S'appuyant I'un sur l'autre, ils donnent lieu à des modèles qui sont ici saisis dans la différence entre cure et entretien. Au terme de ces modèles, apparaît la clinique du narcissisme dont «le caractère polysémique du terme (narcissisme primaire et secondaire, phallique, délibidinalisation, etc.) risque d'en faire également un concept passe-partout. II sera toujours possible de parler d'une inflation ou d'une faille narcissique. Plus intéressant sans doute est le souci d'explorer l'aspect négatif du narcissisme, c'est-à-dire la pauvreté des investissement libidinaux que l'on peut assez faci- 